\title{
Amplification of W-band multi-frequency signals using a gyro-TWA
}

\author{
W. He ${ }^{1}$, C. R. Donaldson ${ }^{1}$, L. Zhang ${ }^{1}$, P. Cain ${ }^{2}$, H. Yin ${ }^{1}$, K. Ronald ${ }^{1}$, \\ A. W. Cross ${ }^{1}$ and A. D. R. Phelps ${ }^{1}$ \\ ${ }^{1}$ Department of Physics, SUPA, University of Strathclyde, Glasgow G4 0NG, Scotland, UK \\ ${ }^{2}$ Keysight Technologies UK Ltd, Edinburgh EH12 9DJ, Scotland, UK
}

\begin{abstract}
A W-band gyrotron traveling wave amplifier (gyroTWA) based on a helically corrugated interaction region achieved an unsaturated output power of $3.4 \mathrm{~kW}$ with a gain of up to $37 \mathrm{~dB}$ and a $3 \mathrm{~dB}$ frequency bandwidth of at least $5.5 \mathrm{GHz}$. In the experiments it was found that the gyro-TWA can be operated with a range of different electron beams. Also the frequency agility of the gyro-TWA was demonstrated by the amplification of multi-frequency signals.
\end{abstract}

\section{INTRODUCTION}

$\mathrm{G}$ yro-devices in the form of both a gyrotron traveling wave amplifier (gyro-TWA) [1] and a gyrotron backward wave oscillator [2] have been developed at the University of Strathclyde. An existing W-band gyro-TWA has achieved an output power of $3.4 \mathrm{~kW}$ with a $3 \mathrm{~dB}$ frequency bandwidth of 91-96.5 GHz (limited by the bandwidth of the input signal) and an unsaturated gain of $37 \mathrm{~dB}$ [3]. The capability of the broad frequency bandwidth is due to the use of a helically corrugated interaction region (HCIR). An "ideal" eigenwave having an almost constant value of group velocity in the designed frequency band is formed in the HCIR to match the dispersion line of the 2 nd harmonic of the electron cyclotron mode allowing broadband amplification [4].

Previously an electron beam of $55 \mathrm{keV}, 1.5 \mathrm{~A}$ with an alpha of $\sim 1.0$ was used. To demonstrate the versatility in operating the gyro-TWA the operating beam voltage was lowered to 40 $\mathrm{kV}$. The $\alpha$ value (the ratio of the transverse velocity to the axial velocity, $\mathrm{v}_{\mathrm{t}} / \mathrm{v}_{\mathrm{z}}$ ) of the electron beam was also reduced to $\sim 0.85$ in order to match the dispersion of the operating eigenwave. The required magnetic field was accordingly adjusted to maintain the resonance between the electron beam mode and the eigenwave. For comparison, the dispersion of the coupling $\mathrm{TE}_{21}$ and $\mathrm{TE}_{11}$ modes, the resultant operating eigenwave and the electron beams in the two experiments are shown in Fig. 1.

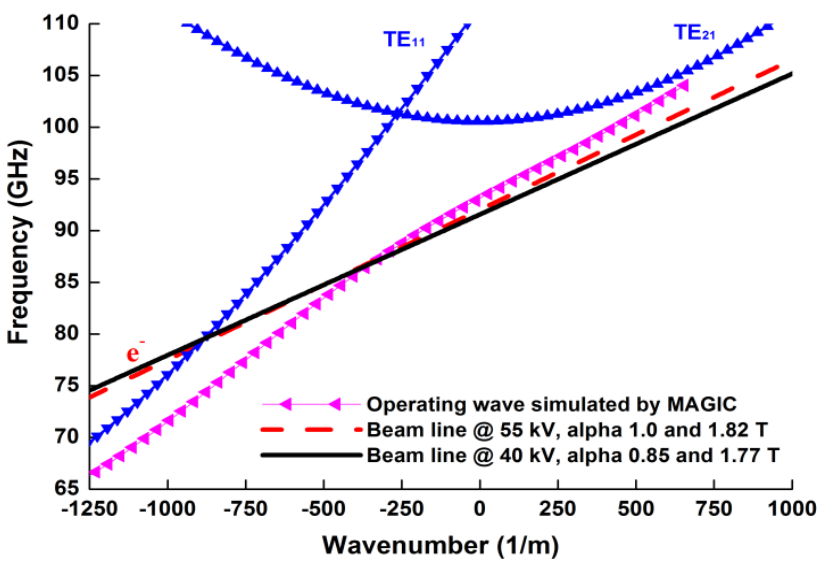

Fig. 1 Dispersion of the operating eigenwave and the electron beams in two separate experiments.
The particle-in-cell (PIC) simulations with the new parameter sets were performed using the 3D PIC code MAGIC and the simulation results are shown in Fig. 2. A gain of about $31 \mathrm{~dB}$ was predicted in the frequency range of $93-94 \mathrm{GHz}$, assuming an alpha spread of $10 \%$ and a beam thickness of 0.1 $\mathrm{mm}$. The simulated gain for the two sets of the parameters that were used in these experiments is shown in Fig. 2 for comparison.

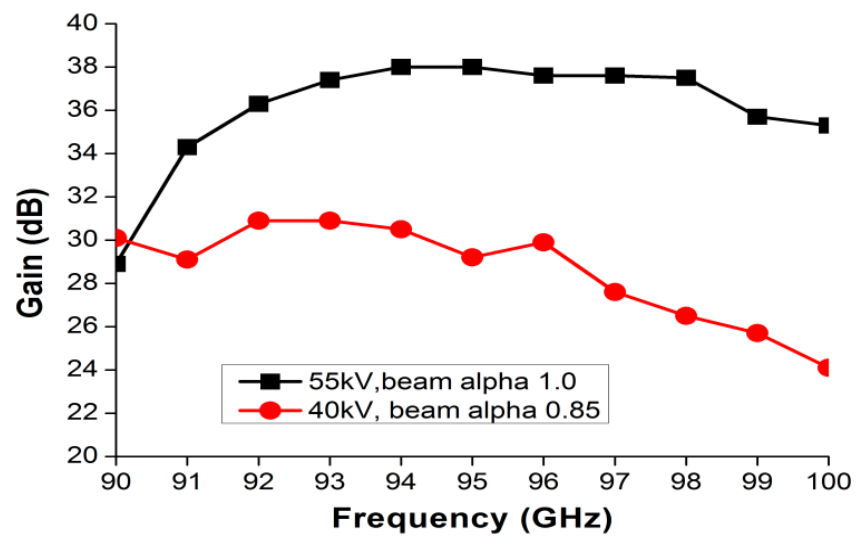

Fig. 2 Simulated gains from the two sets of beam parameters used in the separate experiments.

To demonstrate the promising applications of the gyroTWA in areas such as RADAR, telecommunications, plasma diagnostics and electron spin resonance, the frequency agility of the gyro-TWA was demonstrated by the amplification of a multi-frequency input signal. In the experiment a signal of $\sim 0.5 \mathrm{~W}$, with linearly swept frequency bandwidth of $1 \mathrm{GHz}$, or multi-frequency of $30 \mathrm{MHz}$ separation, was amplified and a gain of over $30 \mathrm{~dB}$ was measured [5].

\section{EXPERIMENT}

The gyro-TWA contained key components, including cusp electron gun [6-8], pillbox window [9], input coupler [10-12], elliptical polarizer [13], output mode converter and windows [14-17], HCIR, and solenoid systems. The axis-encircling electron beam in the gyro-TWA was generated by a cusp electron gun with a magnetic field reversal immediately in front of the cathode. Its beam velocity ratio, $\alpha$, could be tuned by changing the magnetic field at the cathode surface through varying the driving currents of the solenoids. All the mmwave components were optimized through numerical simulations and verified by measurement using a vector network analyzer before and after they were assembled, or vacuum brazed together.

A schematic drawing showing the components of the gyroTWA experiment is shown in Fig. 3. 


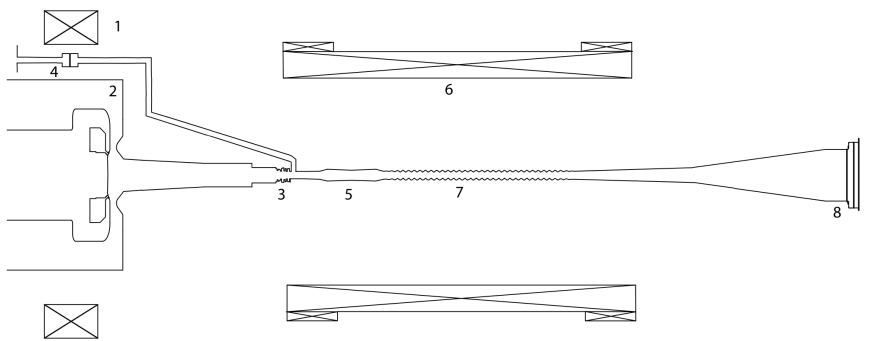

Fig. 3 A schematic drawing of the mm-wave gyro-TWA. (1, 6 - coils, 2 - cusp electron gun, 3 - Bragg reflector, 4 - input coupler, 5 - Elliptical converter, 7 . HCIR, 8 - Corrugated mode converter and output window).

A photo of the upgrade of the frequency-modulated input $\mathrm{mm}$-wave signal is shown in Fig. 1 . An arbitrary waveform generator (model Keysight M8190A (1)) was used to generate signals with a maximum analog bandwidth of $5 \mathrm{GHz}$. The signal was then mixed with a $\sim 15.5 \mathrm{GHz}$ signal from a vector signal generator (model Agilent E8267D (2)). The resultant signal was then used to drive a Quinstar solid-state amplifier (3) to generate a microwave signal in the frequency range of 90 to $96 \mathrm{GHz}$. The signal was attenuated by an Anritsu rotary vane attenuator (4) and measured by a waveguide harmonic mixer (5). The waveform can be processed by an oscilloscope (6) or a signal analyzer (7).

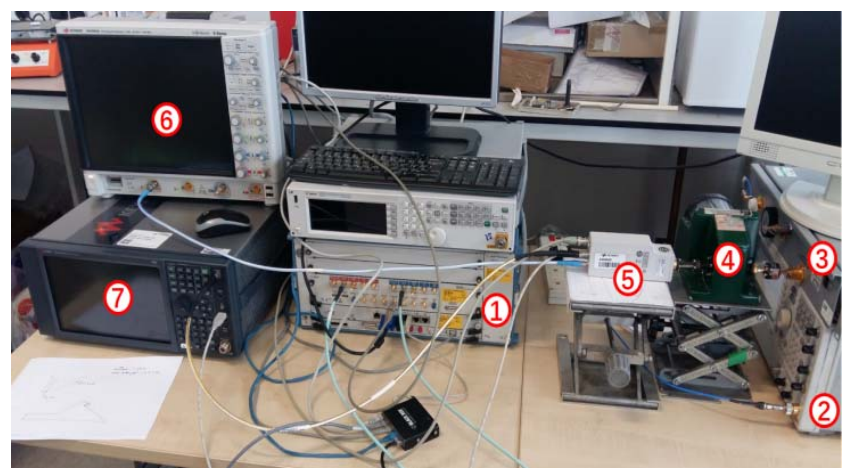

Fig. $4 \mathrm{~A}$ photo showing the setup for generation of the input mm-wave signal.
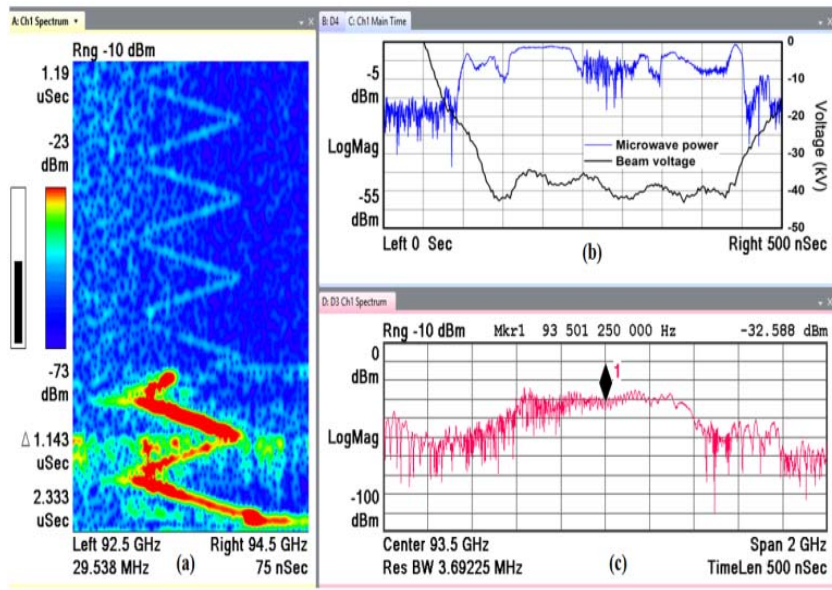

Fig. 5 Gyro-TWA experimental results with frequency-swept signal.

The frequency-swept signal was set as $1.0 \mathrm{GHz}$ bandwidth at the central frequency of $93.5 \mathrm{GHz}$. It can be seen in Fig. 5 (a) that the amplified signal had frequency sweeping that aligned well with the input signal. The correlated microwave signal also matched well with the pulse length of the beam voltage in Fig. 5(b). The amplitude changes were due to variation of the beam voltage. The spectrum of the amplified microwave signal in Fig. 5(c) showed a bandwidth $1 \mathrm{GHz}$.

\section{ACKNOWLEDGMENTS}

The authors thank the EPSRC and STFC UK for supporting this work and Dr. P. Huggard, Mr M. Beardsley and Mr. P. Hiscock of the Millimetre Wave Technology Group at the STFC Rutherford Appleton Laboratory, UK for the construction of the HCIR.

\section{REFERENCES}

[1] G. G. Denisov, V. L. Bratman, A. W. Cross, W. He, et al, "Gyrotron traveling wave amplifier with a helical interaction waveguide," Phys. Rev. Lett., vol. 81, (25), pp. 5680-5683, Dec. 1998.

[2] W. He, C.R. Donaldson, L. Zhang, K. Ronald, et al., "High power wideband gyrotron backward wave oscillator operating towards the terahertz region," Phys. Rev. Lett., vol. 110, (16), art. no. 165101, April 2013.

[3] W. He, C. R. Donaldson, L. Zhang, et al, "Broadband Amplification of Low-Terahertz Signals Using Axis-Encircling Electrons in a Helically Corrugated Interaction Region," Phys. Rev. Lett., vol. 119, (18), art. no. 184801 , Oct. 2017.

[4] V. L. Bratman, A. W. Cross, G. G. Denisov, W. He, et al, "High-gain wide-band gyrotron traveling wave amplifier with a helically corrugated waveguide," Phys. Rev. Lett., vol. 84, (12), pp. 2746-2749, March 2000.

[5] L. Zhang, C. R. Donaldson, P. Cain, A. W. Cross, and W. He, "Amplification of frequency-swept signals in a W-band gyrotron travelling wave amplifier," IEEE Electron Device Lett., pp. 1-4, 2018. DOI: 10.1109/LED.2018.2836868.

[6] C. R. Donaldson, W. He, A. W. Cross, F. Li, A. D. R. Phelps, L. Zhang, K. Ronald, C. W. Robertson, C. G. Whyte, and A. R. Young, "A cusp electron gun for millimeter wave gyrodevices," Appl. Phys. Lett., vol. 96, no. 14, 141501, 2010. DOI: 10.1063/1.3374888.

[7] L. Zhang, W. He, C. R. Donaldson, and A. W. Cross, "Investigation on the optimal magnetic field of a cusp electron gun for a W-band gyro-TWA," Phys. Plasmas, vol. 25, no. 5, 053104, 2018. DOI: 10.1063/1.5027070.

[8] L. Zhang, C. R. Donaldson, and W. He, "Optimization of a triode-type cusp electron gun for a W-band gyro-TWA," Phys. Plasmas, vol. 25, 043120, 2018.

[9] L. Zhang, C. R. Donaldson, A. W. Cross, and W. He, et al., "A pillbox window with impedance matching sections for a W-band gyro-TWA," IEEE Trans, Electron Device. Lett., DOI: 10.1109/LED.2018.2834859.

[10] L. Zhang, W. He, C.R. Donaldson, et al., "Design and measurement of a broadband sidewall coupler for a W-band gyro-TWA," IEEE Trans. Microw. Theory Techn., vol. 63, pp. 3183-3190, 2015.

[11] J. R. Garner, L. Zhang, C. R. Donaldson, A. W. Cross, and W. He, "Design study of a fundamental mode input coupler for a $372-\mathrm{GHz}$ gyroTWA I: Rectangular-to-circular coupling methods," IEEE Trans. Electron Devices, vol. 63, no. 1, pp. 497-503, Jan. 2016.

[12] J. R. Garner, L. Zhang, C. R. Donaldson, A. W. Cross, and W. He, "Design Study of a 372-GHz High Order Mode Input Coupler," IEEE Trans. Electron Devices, vol. 63, no. 8, pp. 3284-3290, Aug. 2016.

[13] L. Zhang, C. R Donaldson and W. He, "Design and measurement of a polarization convertor based on a truncated circular waveguide", J. Phys. D: Appl. Phys., vol. 45, 345103, 2012.

[14] P. McElhinney, C. Donaldson, J. McKay, L. Zhang, D. Robertson, R. Hunter, G. Smith, W. He, \& A. Cross, "An output coupler for a W-band high power wideband gyro-amplifier", IEEE Trans. Electron Devices, vol. 64, no. 1, pp. 1763-1766, 2017.

[15] L. Zhang, W. He, C. R. Donaldson, G. M. Smith, D. A. Robertson, R. I. Hunter, and A. W. Cross, "Optimization and measurement of a smoothly profiled horn for a W-band gyro-TWA", IEEE Trans. Electron Devices, vol. 64, pp. 2665-2669, June 2017. DOI: 10.1109/TED.2017.2687949.

[16] C.R. Donaldson, W. He, L. Zhang, and A.W. Cross, "A W-band mutlilayer microwave window for pulsed operation of gyro-devices," IEEE Micro. Wireless Compon. Lett., vol. 23, pp. 237-239, 2013.

[17] C. R. Donaldson, P. McElhinney, L. Zhang, and W. He, "Wide-band HE11 mode terahertz wave windows for gyro-amplifiers," IEEE Trans. THz Sci. Technol., vol. 6, pp. 108-112, Jan. 2016. 